\title{
Zur Herkunft der deutschen Dialekte in Nordmähren
}

\author{
Mojmír Muzikant
}

The article deals with the phonetics in ten selected German dialects in the area of North Moravia. Discovered dialectal features confirm the mixed language character of the examined area. The South-eastern part of North Moravia is under the influence of the eastern-centreGerman language area. In the central part, the mountain-German dialectal features increase. In the north-west tip of North Moravia, eastern-centre-German dialectal features prevail again.

German dialects in North Moravia - exploration of dialectal speakers living in North Moravia - mixed character of examined area - east-centre-German and Upper German dialectal features

Im vorliegenden Aufsatz wird der ermittelte Lautstand von zehn Basisdialekten Nordmährens im Hinblick auf die Provenienz der festgestellten mundartlichen Elemente klassifiziert. Die Ergebnisse bestätigen den gemischten Charakter des untersuchten Territoriums. Der Südosten Nordmährens steht unter dem Einfluss des Ostmitteldeutschen. Im zentralen Gebiet nehmen oberdeutsche Merkmale zu. Im nordwestlichen Zipfel Nordmährens überwiegen wieder ostmitteldeutsche dialektale Züge.

Deutsche Dialekte in Nordmähren - Erhebungen von Dialektdaten unter der deutschen Restbevölkerung - direkte Methode - gemischter Sprachcharakter des Territoriums ostmitteldeutsche und oberdeutsche mundartliche Züge

\section{Einleitung}

Masařík (1985: 113-117) stellt in seinen Untersuchungen zur mittelalterlichen Geschäftssprache in Mähren fest, dass diese eindeutig einen gemischten Charakter aufweist. Ausgehend von dieser Tatsache, die durch eine ausführliche Analyse des kanzleisprachlichen Materials erhärtet wird, unterteilt er die mittelalterliche Kanzleisprache in drei Gebiete oder „Komplexe“ (Masař́k 1985: 113-114): Den südlichen Komplex bildet das Territorium Südmährens mit den Zentren Nikolsburg, Znaim, Lundenburg. Die eigentliche Sphäre des gemischten bairisch-mitteldeutschen Typs bildet das Territorium von Brünn bis Iglau. Für unsere folgenden Ausführungen ist der dritte Komplex von Belang, und zwar das Gebiet Nordmährens, das jedoch Masařík zufolge weitere Differenzierungen erforderlich macht. Es hat sich gezeigt, dass sich mitteldeutsche (md.) und bairische (bair.) Schichten in Nordmähren sehr oft überlagern, sodass man kaum schärfere Grenzen aufstellen kann (Masař́k 1985: 116). Schwarz (1962) befasst sich u. a. mit der Geschichte der Besiedlung der Sudetenländer durch deutsche Kolonisten, in unserem Fall mit der Besiedlung Nordmährens. Anhand einer Analyse des mundartlichen Sprachmaterials versucht 
er die Herkunft der deutschen Siedler zu erschließen. Er kommt zu dem Schluss, dass die in Nordmähren gesprochenen Dialekte auf eine im westlichen Erzgebirge angesiedelte Bergmannssprache zurückgeführt werden können, die ihrem Wesen nach nordbairische, ostfränkische und mittelbairische Elemente aufweist und überall in Nordmähren Spuren hinterlassen hat (Schwarz 1962: 274).

Bei der Überlegung, ob die Untersuchung mittelalterlicher Kanzleitexte oder eher der gesprochenen Dialekte aussagekräftige Belege in Bezug auf die Herkunft der deutschen Siedler bringen kann, scheint uns die zweite Herangehensweise angemessener zu sein. Selbst Masaŕík gesteht zu (1985: 117), dass sich das Geschriebene nicht nach der Aussprache in den Mundarten (grob Mundartliches wird gemieden), sondern „nach oftmals übergreifenden Schreibtraditionen“ richtet.

Im Gegensatz zu Abhandlungen von E. Schwarz über die sudetendeutschen Mundarten, die v. a. in der Zwischenkriegszeit entstanden sind, steht uns für die Erhebungen von Sprachmaterial lediglich die Restbevölkerung zur Verfügung. Die sehr kleine Zahl der in Nordmähren befindlichen Gewährspersonen (GP) lässt aus vielen Gründen, auf die hier nicht näher eingegangen wird, etliche Zweifel hinsichtlich der Glaubwürdigkeit des eventuell ermittelten mundartlichen Lautstandes aufkommen. Wir haben das Risiko trotzdem eingegangen. Die erforderlichen Sprachdaten zur Untersuchung der Dialekte wurden in direkter Methode gewonnen. Der erfragte Wortschatz deckt die Hauptbereiche des Lebens auf einem Bauernhof $a b$, angefangen von der Nutztierhaltung über den Getreideanbau und die Ernte bis zum Hausbau und Aufbau eines Fuhrwerks. Die Notierungen der Antworten von GP erfolgten im Transkriptionssystem Teuthonista. Insgesamt sind Erhebungen in zehn Ortschaften durchgeführt worden. In der Richtung von Westen nach Osten sind dies Johannesthal/Janov, Engelsberg/Andělská Hora, Klein Stohl/Malá Štáhle, Spachendorf/Leskovec nad Moravicí, Alt Lublitz/Staré Lublice, Domstadtl/Domašov nad Bystřicí, Bodenstadt/Potštát, Daub/Dub, Engelswald/ Mošnov und Senftleben/Ženklava. Im Folgenden werden in Kürze die Ergebnisse vorgestellt.

\section{Gemeinsame Kennzeichen}

In den untersuchten Dialekten gibt es ein ganzes Bündel mundartlicher Elemente, die für alle oder wenigstens für die überwiegende Zahl der untersuchten Entitäten gemeinsam sind. Ein bleibendes Kennzeichen aller untersuchten Dialekte ist der Wandel e > a: Vag „Weg“, a n „essen“, $s$ ä „sehen“ usw. Man kann jedoch Schwankungen in der Häufigkeit beobachten. In Klein Stohl/Malá Štáhle ist eine mäßige Zunahme der e-Belege zu verzeichnen (z. B. me N N „melken“). In Daub/Dub überwiegen die e-Belege (dr e v n „treffen“, aber: $d r$ a z n „dreschen“). Im Gegensatz 
dazu sind a-Belege in Senftleben/Ženklava in sehr großem Maß vertreten, so z. B.

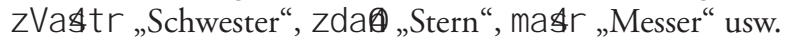

Umgekehrt ist der Wandel i > e nicht in Klein Stohl/Malá Štáhle und Daub/Dub vertreten. Die Erklärung für das Fehlen dieses Merkmals in Daub/Dub ist auf die starke Nivelierung des Dialekts bzw. des Idiolekts der Sprecherin zurückzuführen. Der Wandel gehört ja zu den omd. Kennzeichen. In den übrigen Ortsdialekten kommt die Senkung i > e vor, wobei sie in großer Zahl in Jahnsdorf/Janov (me \& „Mist“, de z | r „Tischler“, g He A „Kinn“), Engelsberg/Andělská Hora (Ve „Wiese“, g He z|e „Schlüssel“, z Ve

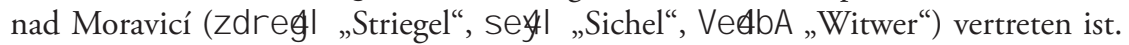
Nur selten ist der Wandel dagegen in Alt Lublitz/Staré Lublice zu verzeichnen (Ve „Wiese“, aberd i Ez „Tisch“, z i 日nben „Schienbein“) usw.

Diphthongierungen kennen wiederum Senftleben/Ženklava und Engelsberg/ Andělská Hora nicht. Dagegen ist die Diphthongierung sehr stark in Klein Stohl/ Malá Štáhle (Voug „Waage“, nuEs „Nuß“) und in Spachendorf/Leskovec nad

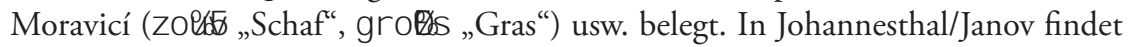
sich diese Erscheinung nur selten (mo Bn „Mann“, z e日nA dog „schöner Tag“) usw. Die Senkung u > o vor Nasal ist in Daub/Dub, Jahnsdorf/Janov, Alt-Lublitz/Staré Lublice und zum Teil auch in Senfleben/Ženklava anzutreffen. In allen anderen Ortschaften bleibt u vor Nasal erhalten, so z . B. in Klein Stohl/Malá Štáhle: di s ün „die Sonne“, s ümA „Sommer“ usw.

\section{Spezifisches in den untersuchten Dialekten}

Vor der Einordnung der aufgezeichneten Merkmale werden hier die lautlichen Besonderheiten der jeweiligen Basisdialekte aufgezählt. Zuerst führen wir solche Merkmale an, durch die sich die einzelnen Dialekte auf dem untersuchten Territorium voneinander unterscheiden.

Für Engelswald/Mošnov ist Folgendes kennzeichnend: Vokalisierung des Vibranten vor Konsonant: g Ho A 5 Korn“, Voranstellung (Einschub) eines Halbvokals vor Vibranten bei Einsilbern: $\mathrm{C} O \boldsymbol{\theta} \bar{B}$ „Rohr“, b > V g e z d u i 5 e 5, gestorben“, Infinitive auf e $\bar{b} \mathrm{~d}$ r a e $\$$,,treffen“. In Jahnsdorf/Janov begegnet man dem Wandel o/0 ozu u vor Vibranten: g Hur b „Korb“, du 0 „Tor“. Als Infinitivendung wird konsequent abgeschwächter Vokal -A gebraucht: I a A „lesen“, he $\mathbf{B}$ | A „heilen“. Die Apokope bleibt aus. Alt-Lublitz/Staré Lublice fällt nur durch vereinzelte Rundungen auf: g Hü r y „Kirche“. In Engelsberg/Andělská Hora wird ähnlich wie in Klein Stohl/ Malá Štáhle der Liquid l vor Konsonant und im Auslaut vokalisiert: g Ho i 6 „Kalb“, Z d o \%5,Stall“. Auch der Wegfall des Liquids vor Konsonant und im Auslaut sowie auch Labialisierungen sind hier gleich wie in Klein Stohl/Malá Štáhle bekannt, im letzteren Ort jedoch noch in größerem Ausmaß vorhanden: gö đ „Geld“, z nö日 
„schnell“, gö̈ „gelb“, bü日 „Bild“. Der Frikativ V wird beim Pronomen 'wir'zum labialen Verschlusslaut: b ë e $5 \mathrm{~g}$ Hu me 5, wir kommen“. Mhd. ei wir konsequent zu a $\theta$ VaAsnK,Weizen“.

Demgegenüber bleiben Liquide in Senftleben/Ženklava erhalten, allerdings werden sie oft velarisiert: go $\mathrm{Bl}$ l 2,"Gabel“. Die Velarisierung betrifft, und diese Erscheinung ist nur in diesem Ort belegt, Nasale vor Dentalen im Inlaut: ve Nde 5, finden“, hu Nd „Hund“. Der Verschlusslaut b wird ähnlich wie in Engelswald/Mošnov zum stimmhaften Frikativ: vuOAr i Ve 5,Futterrübe“. Zu den weiteren Besonderheiten

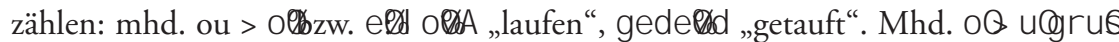

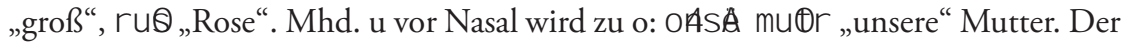
Wandel e $0>$ i Obleibt aus. In Domstadtl/Domašov nad Bystřicí werden Liquide vor Konsonant im Gegensatz zu Engelsberg/Andělská Hora und Klein Stohl/Malá Štáhle nur zum Teil vokalisiert: go i b „Kalb“, aber: g Ho I Ó „kalt“. Der labiale Frikativ V beim Pronomen 'wir' wird häufig ähnlich wie in Engelsberg/Andělská Hora zum stimmhaften Verschlusslaut: bëAgals a A $\$$ „Wir geben es euch“. Bodenstadt/ Potštát fällt durch keine spezifischen Lautentwicklungen auf, die woanders nicht zu belegen wären. Spachendorf/Leskovec nad Moravicí zeichnet sich durch eine auffällige Vielzahl an Diphthongierungen aus. Nicht nur die mhd. Vokale e, e 0 o, 0 l sondern auch verdumpftes a und die hohen Vokale u und i werden diphthongiert:

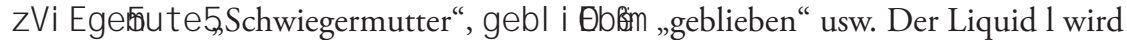
vor Konsonant ähnlich wie in Engelsberg/Andělská Hora, Klein Stohl/Malá Štáhle und Domstadtl/Domašov nad Bystřicí, jedoch nur in geringerem Maße, vokalisiert. h 0 i 6 „holen“, aber: hol ds „,Holz“. In Einzelfällen fällt l aus: v E z bi $\theta$ „verspielen“, aber: $z d a \theta n$ „stehlen“.

\section{Herkunft der festgestellten mundartlichen Elemente}

Bei der Bestimmung der Herkunft der festgestellten mundartlichen Elemente, die auch bei der Bestimmung der Herkunft der deutschen Kolonisten in Nordmähren eine große Rolle spielen, gehen wir von unseren früher in Süd- und Mittelmähren durchgeführten Erhebungen sowie auch von Jungandreas (1937: 483, 505) aus, der anhand des v.a. schriftlichen Materials anführt, dass in Nordmähren neben dem Ostmitteldeutschen (Omd.) auch mit starkem bairischem Einfluss zu rechnen ist. Die mundartlichen Elemente können wir deshalb unter Anlehnung an Jungandreas sowie an unsere Erkenntnisse in schlesisch/omd. und oberdeutsche (obd.) Züge einteilen (s. die unten angeführte Tabelle). 


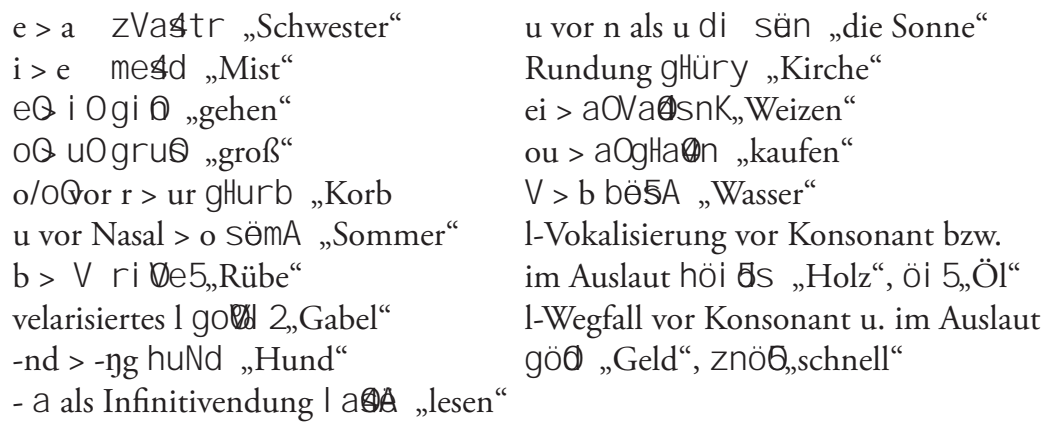

Nicht berücksichtigt werden einige omd. Erscheinungen wie z. B. -p statt mhd. -pf und die Kontraktionen -age-, -ege- usw. Ohne eine eindeutige Zuordnung bleiben vorläufig Diphthongierungen von mhd. o/ 00 e/e $0 \mathrm{i}$, u und verdumpftem a/a 0 Sie sind nämlich sowohl in schl. als auch in bairischen Dialektgebieten überliefert, vgl. Jungandreas (1937: $\$ \$ 78,80,134,162,168,178)$. Masaŕík (1985: 32-34) verzeichnet nur vereinzelt isolierte Fälle, die als Diphthongierungen des mhd ë bzw. i vor $\mathrm{r}$ zu interpretieren sind. In unserem mundartlichen Material aus der Brünner und Wischauer Sprachinsel (SI) sind dagegen Diphthongierungen häufig anzutreffen. Im vorgestellten mundartlichen Material aus Nordmähren sind Diphthongierungen in zwei Ortschaften gar nicht vorhanden: in Senftleben/ Ženklava und in Engelsberg/Andělská Hora. Die eine Ortschaft zeichnet sich dabei durch einen ausgeprägten omd. Charakter aus, in der anderen sind sowohl obd. als auch omd. Züge regelmäßig vertreten. Diese Tatsache unterstützt die These, dass Diphthongierungen als klares Kriterium für die Unterscheidung von omd. und obd. Zügen kaum herangezogen werden können.

In Bezug auf die Merkmale, die für die Einteilung der Dialekte herangezogen werden können, stellen wir uns folgende Fragen: Welche von den untersuchten Ortschaften sind ausgeprägt omd. und welche obd.? Welche der Ortschaften sind sprachlich vermischt? Welche territorialen Elemente überwiegen? Welche sind im Hintergrund? Gibt es auch in einem Ort gleichmäßig verteilte, unterschiedliche räumliche Einflüsse? Nach einer gründlichen Abwägung der omd. und obd. Elemente lässt sich Folgendes zum Sprachcharakter der untersuchten Ortschaften sagen (Abb. 1):

Daub/Dub ist sehr ausgeglichen; vorhanden sind nur Diphthongierungen und erhaltenes u vor Nasal. Eine Zuordnung ist schwer durchführbar. Klein Stohl/Malá Štáhle zeichnet sich durch eine gleichmäßige Vermischung von obd. und omd. Sprachelementen aus. In Engelswald/Mošnov überwiegen eindeutig omd. Elemente. 
Ebenfalls in Johannesthal/Janov sind omd. Sprachelemente im Vordergrund, die ausgeprägt dialektalen Merkmale sind aber zurückgegangen. Ein Rückgang mundartlicher Elemente ist auch in Alt Lublitz/Staré Lublice sichtbar. Wenn die Diphthongierungen mit einbezogen werden, sind die obd. Merkmale mäßig im Übergewicht. Engelsberg/Andělská Hora steht ganz eindeutig unter dem Einfluss des Obd. Der Ortsdialekt in Senftleben/Ženklava trägt einen ausgeprägt omd./schl. Charakter. Obd. ist praktisch nicht belegt. In Domstadtl/Domašov nad Bystřicí gewinnen obd. Elemente an Boden, die omd. Merkmale stehen im Hintergrund. In Bodenstadt/Potštát nehmen mundartliche Elemente im allgemeinen ab, von den überlieferten überwiegen stark die obd. In Spachendorf/Leskovec nad Moravicí sind Erscheinungen beider Provenienzen häufig vertreten, sie sind im Gleichgewicht.

Zusammenfassend kann anhand der beigefügten Abbildung, Sprachcharakter der untersuchten Ortschaften' bezüglich der räumlichen Verteilung der mundartlichen Elemente ergänzt werden, dass der Osten Nordmährens, vertreten durch Engelswald/ Mošnov und Senftleben/Ženklava, unter eindeutigem Einfluss des Omd. steht. Im zentralen Teil Nordmährens nehmen die obd. Bestandteile zu, in vier Ortschaften bilden sie sogar die Mehrheit der belegten mundartlichen Elemente: Bodenstadt/ Potštát, Domstadtl/Domašov nad Bystřicí, Alt Lublitz/Staré Lublice, Engelsberg/ Andělská Hora. Im nordwestlichen Zipfel Nordmährens, vertreten durch Johannesthal/Janov, überwiegen wiederum omd. dialektale Züge.

Abb. 1: Sprachcharakter der untersuchten Ortschaften in Nordmähren (Mojmír Muzikant)

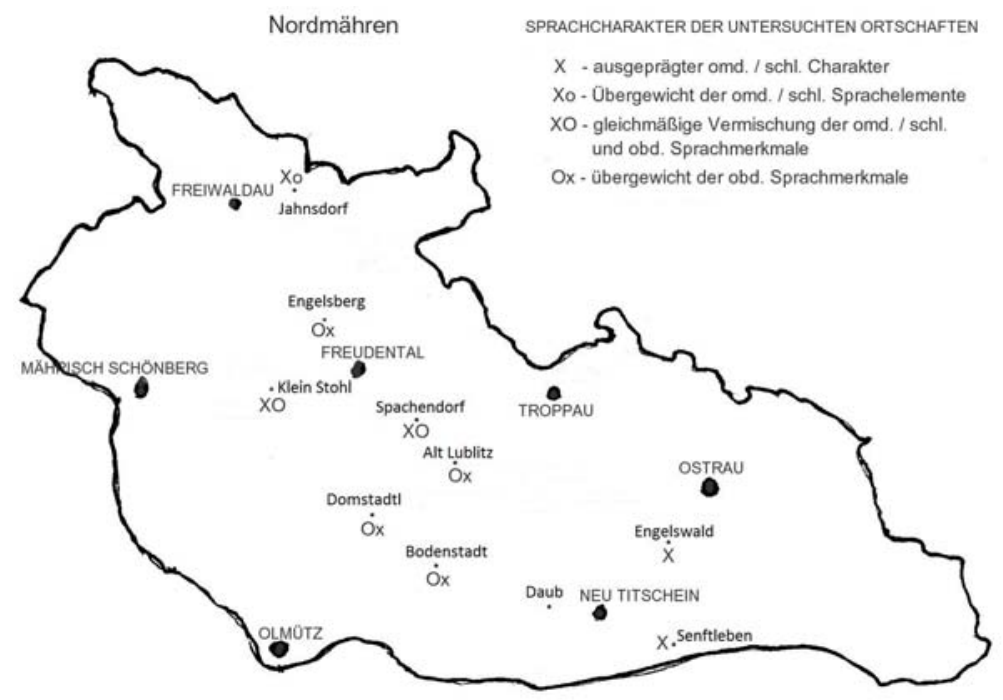




\section{Literaturverzeichnis}

Jungandreas, Wolfgang (1937): Zur Geschichte der schlesischen Mundart im Mittelalter. Breslau: Maruschke und Berendt.

Kranzmayer, Eberhard (1956): Historische Lautgeographie des gesamtbairischen Dialektraumes. Wien: H. Böhlaus Nachf.

Masařík, Zdeněk 1985: Die frühneuhochdeutsche Geschäftssprache in Mähren. Brno: MasarykUniversität.

Muzikant, Mojmír (2007): Der Sprachatlas der deutschen Mundarten in Tschechien, vorläufige Forschungsresultate des tschechischen Teilteams und Perspektiven. In: Muzikant, Mojmír (Hg.): Deutsche Dialekte in Tschechien, ihre Gemeinsamkeiten und Besonderheiten im Vergleich zum Mutterboden. Brno: Masaryk-Universität. S. 7-16.

Schwarz, Ernst (1962): Sudetendeutsche Sprachräume. München: Lerche. 